\title{
Transliteration and Abbreviation
}

In transliterating ancient Greek, we have thought it reasonable, if not entirely consistent, to use a Latinized spelling for words or proper names for which this has become "normal" English usage and in other cases to use a direct transliteration from the Greek. In some instances, such as the nomenclature of clothing or types of Greek vases, the system is unavoidably inconsistent, as it depends more or less on the frequency with which these words have been used in English (e.g., chiton and krokotos; cantharus and khous). A similar inconsistency occurs in our rendition of the epithets and alternate names of the god Dionysus himself. Some of his more familiar names, well known to us from the Roman poets, appear in their familiar Latin form (e.g., Bromius or Bacchus), while rarer, epichoric forms (e.g., Meilikhios, Bakkheus, or Omestes) have been directly transliterated from the Greek.

In hope of making the citation of ancient sources clearer to the nonspecialist, we have devised the following two-tiered method of reference: (I) the titles of better-known authors are cited either in full by their English titles (e.g., Aeschylus Seven against Thebes, Sophocles Oedipus at Colonus) or by easily decipherable abbreviations (e.g., Aesch. Seven or Soph. Oed. Col.); (2) the works of more obscure authors are always cited in full and usually by their Latin titles (e.g., Philostratus Imagines). Most references to modern secondary literature consist of author's name and the date of publication; full references can 
be found to these works in the bibliography at the end of the volume. We have limited our use of abbreviations to the following collections, dictionaries, and frequently used journals (please note that series or collections that are listed below without the name of an editor are the work of many hands).

\section{Collections of Artifacts or Images}

$\begin{array}{ll}A B L & \text { C. H. E. Haspels. Attic Black-Figure Lekythoi. Paris, I936. } \\ \text { ABV } & \text { J. D. Beazley. Attic Black-Figure Vase-Painters. Oxford, I956. } \\ \text { ANRW } & \text { Aufstieg und Niedergang der römischen Welt. Berlin, 1972-. } \\ \text { ARV } 2 & \text { J. D. Beazley. Attic Red-Figure Vase-Painters. 2d ed. Oxford, } \\ & \text { I963. } \\ \text { CSE } & \text { Corpus speculorum Etruscorum. } \\ C V A & \text { Corpus vasorum antiquorum. } \\ \text { ES } & \text { B. Gerhard, A. Klugmann, and G. Korte. Etruskische Spiegel. } \\ \text { Gens antiq } & \text { Berlin, I840-97. } \\ \text { Gens antiquissima Italiae: Antichità dall 'Umbria a Leningrado. Pe- } & \text { rugia. }\end{array}$

\section{Collections of Inscriptions}

CIE

CIG

CIL

CIRB

GV

IG

$I G B R$

IGSK

IGUR

IKlaudiu Polis

$I K \gamma z i k o s$

IMagnesia
Corpus inscriptionum Etruscarum. Leipzig and Rome, 1893present.

Corpus inscriptionum Graecarum. Berlin, I 828-77.

Corpus inscriptionum Latinarum. Berlin, I 863-present.

Corpus inscriptionum Regni-Bosporani. Leningrad, I965.

W. Peek, ed. Griechische Vers-Inschriften. Berlin, I955.

Inscriptiones Graecae. Berlin, I873-present.

G. Mihailov, ed. Inscriptiones Graecae in Bulgaria repertae. Sofia, I956-66.

Inschriften griechischer Städte aus Kleinasien. Bonn, 1972-present.

L. Moretti, ed. Inscriptiones Graecae urbis Romae. Rome, I968present.

Becker-Bertau, ed. Die Inschriften von Klaudiu Polis. IGSK 3 I. Bonn, 1986.

E. Schwertheim, ed. Die Inschriften von Kyzikos und Umgebung. IGSK I 8 and 26. Bonn, 1980-83.

O. Kern, ed. Die Inschriften von Magnesia am Maeander. Berlin, I900. 
INikaia

IPergamon

ISmyrna

LSAM

LSCG

LSS

OGI

SEG

$S I G^{3}$

TAM
S. Sahin, ed. Katalog der antiken Inschriften des Museums von Iznik (Nikaia). Bonn, I979-82.

M. Fraenkel, ed. Die Inschriften von Pergamon. Altertümer von

Pergamon 8. Berlin, I 890-95.

G. Petzl, ed. Die Inschriften von Smyrna. IGSK 23, 24. I, and 24.2. Bonn, 1982-90.

F. Sokolowski, ed. Lois sacrées de l'Asie Mineure. Paris, I955.

-

- ed. Lois sacrées des cités grecques: Supplément. Paris, I962.

W. Dittenberger, ed. Orientis Graeci inscriptiones selectae. Leipzig, I903-5.

Supplementum epigraphicum Graecum. Leiden, I923-present.

W. Dittenberger, ed. Sylloge inscriptionum Graecarum. $3 \mathrm{~d}$ ed. Leipzig, I92 I-24.

Tituli Asiae Minoris. Vienna, I90 I-present.

\section{Collections of Literary Fragments}

\begin{tabular}{|c|c|}
\hline $\begin{array}{l}E G F \\
F G r H\end{array}$ & $\begin{array}{l}\text { M. Davies, ed. Epicorum Graecorum fragmenta. Göttingen, I } 988 . \\
\text { F. Jacoby, ed. Die Fragmente der griechischen Historiker. Berlin } \\
\text { and Leiden, I923-58. }\end{array}$ \\
\hline FHG & $\begin{array}{l}\text { C. Müller and T. Müller, eds. Fragmenta historicorum Graecorum. } \\
\text { Paris, I } 84 \mathrm{I} \text {. }\end{array}$ \\
\hline$F V S$ & $\begin{array}{l}\text { H. Diels and W. Kranz, eds. Die Fragmente der Vorsokratiker. } \\
\text { Berlin, I952. }\end{array}$ \\
\hline Nauck ${ }^{2}$ & $\begin{array}{l}\text { A. Nauck. Tragicorum Graecorum fragmenta. } 2 \mathrm{~d} \text { ed. Leipzig, } \\
\text { I } 889 .\end{array}$ \\
\hline OF & O. Kern, ed. Orphicorum fragmenta. Berlin, I9I2. \\
\hline$P C G$ & $\begin{array}{l}\text { R. Kassel and C. Austin, eds. Poetae comici Graeci. Berlin, I983- } \\
\text { present. }\end{array}$ \\
\hline$P L G$ & T. Bergk, ed. Poetae lyrici Graeci. Leipzig, I 882 . \\
\hline & $\begin{array}{l}\text { D. L. Page, ed. Poetae melici Graeci. Oxtord, } 1902 . \\
\text { H. von Arnim, ed. Stoicorum veterum fragmenta. Leipzig, I903- }\end{array}$ \\
\hline $\operatorname{Tr} G F$ & $\begin{array}{l}\text { B. Snell and S. Radt, eds. Tragicorum Graecorum fragmenta. } \\
\text { Göttingen, I97I-present. }\end{array}$ \\
\hline
\end{tabular}

\section{Collections of Papyri}

PAntinoöpolis C. H. Roberts, J. W. B. Barns, and H. Zilliacus, eds. The Antinoöpolis Papyri. London, 1950-66.

PGurob

J. G. Smyly, ed. Greek Papyri from Gurob. Dublin, I92 I. 
xii Transliteration and Abbreviation

PHerc POxy
M. Gigante, ed. Catalogo dei papiri ercolanesi. Naples, 1979. The Oxyrhynchus Papyri. London, I 898-present.

\section{Dictionaries and Lexica}

$D A$

LIMC

LSJ

$R E$

\section{Journals}

$A A$
$A E$
$A J A$
$A M$

AntK
$A R$
$B C H$
Meded
RA
SBBerlin
ZPE

C. Daremberg and E. Saglio, eds. Dictionnaire des antiquités grecques et romaines. Paris, 1877-1919.

Lexicon iconographicum mythologiae classicae. Zurich, 198Ipresent.

H. G. Liddell, R. Scott, and H. S. Jones, eds. Greek-English Lexicon with a Supplement. 9th ed. Oxford, 1968.

Pauly-Wissowa. Real-Encyclopädie der classischen Altertumswissenschaft. Stuttgart, I894-I980.

Archäologischer Anzeiger.

L'anneé épigraphique.

American Journal of Archaeology.

Mitteilungen des Deutschen Archäologischen Instituts, Athenische Abteilung.

Antike Kunst.

Archaeological Reports.

Bulletin de correspondance hellénique.

Mededeelingen van het Nederlands Historisch Institut te Rome.

Revue archéologique.

Sitzungsberichte der Deutschen Akademie der Wissenschaften zu Berlin, Klasse für Sprachen, Literatur und Kunst.

Zeitschrift für Papyrologie und Epigraphik. 